\title{
Molecular detection of genotypes and subtypes of Cryptosporidium infection in diarrheic calves, lambs, and goat kids from Turkey
}

\section{Mohammad Hazzaz Bin Kabir}

National Research Center for Protozoan Diseases, Obihiro University of Agriculture and Veterinary Medicine, Obihiro, Japan.

\section{Onur Ceylan}

Faculty of Veterinary Medicine, Selcuk University, Konya, Turkey.

\section{Ceylan Ceylan}

Faculty of Veterinary Medicine, Selcuk University, Konya, Turkey.

\section{Ayman Ahmed Shehata}

Laboratory of Sustainable Animal Environment, Graduate School of Agricultural Science, Tohoku University, Japan.

\section{Hironori Bando}

Laboratory of Sustainable Animal Environment, Graduate School of Agricultural Science, Tohoku University, Japan.

\section{Mohamed Ibrahim Essa}

Faculty of Veterinary Medicine, Zagazig University, Egypt.

\section{Xuenan Xuan}

National Research Center for Protozoan Diseases, Obihiro University of Agriculture and Veterinary Medicine, Obihiro, Japan.

\section{Ferda Sevinc}

Faculty of Veterinary Medicine, Selcuk University

Kentaro Kato ( $\nabla$ kentaro.kato.c7@tohoku.ac.jp)

Tohoku University

\section{Research}

Keywords: Calves, C. bovis, C. parvum, Cryptosporidium, Diarrhea, Goat kids, Lambs, Subtypes, Turkey

Posted Date: February 19th, 2020

DOI: https://doi.org/10.21203/rs.2.23993/v1 
License: (c) (i) This work is licensed under a Creative Commons Attribution 4.0 International License. Read Full License

Version of Record: A version of this preprint was published at Parasitology International on December 1st, 2020. See the published version at https://doi.org/10.1016/j.parint.2020.102163. 


\section{Abstract}

Background: Cryptosporidium spp. are enteric protozoan parasites that infect a wide range of animals as well as humans. The studies on Cryptosporidium infections of animals in Turkey are mostly rely on microscopic observation. Few data are available regarding the distribution of Cryptosporidium genotypes and subtypes infection. The aim of this study is to analyse the zoonotic potential of Cryptosporidium oocysts shed from young ruminant livestock.

Methods: A total of 415 diarrheic fecal specimens from 333 calves, 67 lambs, and 15 goat kids were examined for the presence of Cryptosporidium oocysts by microscopy. Microscopic positive specimens were then analyzed for Cryptosporidium genotypes and subtypes detection by use of nested PCR of the small subunit ribosomal RNA (SSU rRNA) gene and the highly polymorphic $60 \mathrm{kDa}$ glycoprotein (gp60) gene followed by sequence analyses.

Results: The results of this study revealed that $25.6 \%$ (106 of 415) of the specimens were positive for Cryptosporidium spp. infection by microscopic examination and molecular analysis. We identified $27.4 \%$ (91/333), $19.4 \%$ (13/67), and 13.4\% (2/15) of positivity in calves, lambs and goat kids, respectively. Genotyping of the SSU rRNA indicated that almost all positive specimens were of $C$. parvum , except for one calf which was of $\mathrm{C}$. bovis. Sequence analysis of the gp60 gene revealed the most common zoonotic subtypes (Ila and Ild) of C . parvum. We detected 11 subtypes (IlaA11G2R1, IlaA11G3R1, IlaA12G3R1, IlaA13G2R1, IlaA13G4R1, IlaA14G1R1, IlaA14G3R1, IlaA15G2R1, IldA16G1, IldA18G1, IIdA22G1); three of them (IlaA12G3R1, IlaA11G3R1 and IlaA13G4R1) was novel subtypes found in calves and lambs. Additionally, three subtypes (IlaA11G2R1, IlaA14G3R1, and IIdA16G1) were detected in calves, lambs, and goat kid for the first time in Turkey.

Conclusions: The findings illustrate the high occurrence of Cryptosporidium infection in Turkey and suggest that calves, lambs, and goat kids are likely a major reservoir of $\mathrm{C}$. parvum and a potential source of zoonotic transmission, which may have public health implications. Keywords: Calves, C. bovis, C. parvum , Cryptosporidium , Diarrhea, Goat kids, Lambs, Subtypes, Turkey.

\section{Background}

Cryptosporidiosis is a primary disease playing role in the etiology of neonatal diarrhea syndrome of ruminants and causes severe illness or death in young animals [1]. Among the 16 recognized species, Cryptosporidium parvum is of medical and veterinary importance. Cryptosporidium parvum has a number of subtypes that can be grouped into subtype families as measured by gp60 sequence analyses [2]. Healthy and diarrheic calves less than one month of age may facilitate the transmission of cryptosporidiosis in both humans and animals [3]. Other livestock are also potential reservoirs of this protozoan. In cattle herds, infected animals, particularly diarrheic calves, may act as sources of direct infection for other livestock [4]. Considering the worldwide distribution and zoonotic relevance of Cryptosporidium spp., a better understanding of transmission pathways and species distribution is 
important for public health [5]. In addition, indirect transmission by cattle sheds, bedding, pasture, and soil and contaminated drinking water from environmental oocysts, has been reported as a major source of bovine transmission [6]. The clinical course of the disease in lambs and goat kids are similar to those of calves.

The distribution of Cryptosporidium species in goat kids has been reported to be similar to those of lambs, with the occurrence of mainly C. parvum, C. xiaoi, and C. ubiquitum [7-13]. However, geographic differences exist in the distribution of Cryptosporidium species in lambs, with C. parvum as the dominant species in European countries, C. ubiquitum in the Americas, C. xiaoi in developing countries, and all three species common in Australia [14]. In addition, European studies revealed that C. parvum was mostly found in clinically affected lambs, whereas C. ubiquitum and C. xiaoi were commonly observed in healthy lambs $[7,15,16]$.

There are a few studies on Cryptosporidium infections in animals in Turkey; however, most of them rely on microscopic observation of oocysts and detection of coproantigens by the ELISA method. Cryptosporidiosis in slaughtered animals was investigated in Van province [17]. Since then, Cryptosporidium oocysts have been reported in animals with and without diarrhea from Kars [18], Erzurum [19], and Van provinces of Turkey [20]. Recently, a study mentioned C. parvum was the predominant species in pre-weaned calves whereas C. bovis and C. ryanae were mostly found in postweaned calves and heifers in the Mediterranean and Central Anatolia regions of Turkey [21]. Another study reported the distribution of $\mathrm{C}$. parvum in free-range pre-weaned diarrheic calves and goat kids in five provinces of Turkey [22]. Two studies subtyped C. parvum-positive isolates from pre-weaned calves by using gp60 sequence analysis in Kars, Turkey $[23,24]$.

Molecular diagnostic approaches make it possible to detect human infections deriving from agricultural animals. Accordingly, the goal of the present study was to assess the zoonotic potential of Cryptosporidium by identifying the genotype and subtype of Cryptosporidium spp. in the diarrheic calves, lambs, and goat kids in Turkey.

\section{Methods}

\section{Specimen collection}

A total of 415 diarrheic fecal specimens from 333 calves, 67 lambs, and 15 goat kids were collected between 2016 and 2018 from Konya province, Turkey. Diarrhea was observed during the time of specimen collection. All relevant data such as age and consistency of diarrhea were recorded at the time of sample collection for further analysis. Each specimen was placed into an individual sterile polystyrene tube with $2.5 \%$ potassium dichromate, labelled, and kept at $4{ }^{\circ} \mathrm{C}$ until DNA extraction.

\section{Specimen analyses}


Specimens were microscopically examined for Cryptosporidium spp. oocysts by use of the modified Ziehl-Neelson (MZN) method [25]. DNA was extracted directly from the specimens by using the Biomasher IV (Funakoshi Co., Ltd.) and NucleoSpin ${ }^{\circledR} T$ issue (MACHEREY-NAGEL) kits according to the manufacturer's instructions [26]. After extraction, DNA was stored at $-20^{\circ} \mathrm{C}$. For genotyping and subtyping of Cryptosporidium detection, an approximately 830-bp fragment of the SSU rRNA gene and the gp60 gene were amplified by using KOD FX Neo (TOYOBO, Japan) with primers as described previously $[27,28]$. For the primary PCR, a product of 1325 bp was amplified using the following primers: 5'- TTCTAGAGCTAATACATGCG -3' and 5'-CCCATTTCCTTCGAAACAGGA-3'. Then, a nested PCR was done using the following primers: 5'-GGAAGGGTTGTATTTATTAGATAAAG-3' and 5'AAGGAGTAAGGAACAACCTCCA -3' to amplify the 830-bp fragment of the Cryptosporidium SSU rRNA gene. Cryptosporidium baileyi genomic DNA and ultrapure water were used as positive and negative controls, respectively, in all PCR sets. The amplified fragments were electrophoresed in $1.5 \%$ agarose, stained with GelRed $\circledast$ (Biotium), and visualized on an UV transilluminator in the QIAxcel Advanced system (Qiagen, Valencia, California). The identity of each Cryptosporidium species was confirmed by sequence analysis of the secondary PCR products from the specimens. The gp60 gene was used to subtype $C$. parvum. The subtypes were named after sequencing of gp60 gene of these isolates based on the number of trinucleotide repeats encoding the amino acid serine (TCA, TCG, TCT, and ACATCA) sequence [29].

\section{Sequencing and phylogenetic analyses}

All secondary PCR products were sequenced in both directions by using an ABI 3130 Genetic Analyzer (Applied Biosystems Japan, Tokyo, Japan) with the secondary primers and the BigDye Terminator v3.1 Cycle Sequencing Kit (Applied Biosystems, Tokyo, Japan). The sequences were aligned by using Clustal $X_{2}$ [30], and the computed sequences were edited by hand with BioEdit 7.0.5.3 [31]. All gaps were eliminated and SSU rRNA genes were used for the phylogenetic analysis. Maximum Likelihood analyses were performed by using MEGA version 7 software program [32]. Substitution models and optional parameter sets were selected according to the Akaike information criterion. To assess the reliability of the tree, bootstrap analysis was done with 1000 replicates using the same datasets. We constructed two phylogenetic trees: one for the SSU rRNA gene, in which the substitution model and optional parameters were also used on the Tamura 3-parameter model [33], and the other for the gp60 gene, in which the Hasegawa-Kishino-Yano model [34] was used, incorporating the invariable site and Gamma distribution options.

\section{Results}

\section{Genotype detection}


Of the 415 fecal specimens investigated, 106 (25.6\%) were confirmed by the MZN technique to be infected with Cryptosporidium spp. oocysts. All microscopically positive fecal specimens were also nested PCR positive. Two Cryptosporidium species, namely $C$. parvum and $C$. bovis were identified in calves using SSU rRNA gene analyses, accounting for $27.1 \%$, and $0.3 \%$, respectively. Only Cryptosporidium parvum was identified in lambs and goat kids using SSU rRNA gene analyses, accounting for $19.4 \%$, and $13.4 \%$, respectively (Table 1 ).

\section{Specimen analyses}

Of the 415 fecal specimens, Cryptosporidium was found more frequently in 3-7-day-old calves (30.8\%) than in 8-15-day-old calves (26.3\%) and 16-30-day-old calves (17.8\%). Cryptosporidium prevalence was also higher in younger lambs: $23.1 \%$ in $3-7$-day-old lambs compared with $14.2 \%$ in both $8-15$-day-old lambs and 16-30-day-old lambs. However, in goat kids, $12.5 \%$ of specimens from $3-7$-day-old animals were Cryptosporidium-positive compared with $16.7 \%$ of specimens from 8-15-day-old animals (Table 2). Most of the specimen contained watery consistency diarrhea, and Cryptosporidium was found predominantly in the watery consistency diarrhea of the calves, lambs, and goat kids (Additional file 1: Table S1).

\section{Phylogenetic analyses}

All PCR-positive specimens were directly sequenced; analyses of the nucleotide sequences of the SSU rRNA genes revealed the presence of $C$. parvum in calves, lambs, and goat kids, and the presence of $C$. bovis in one calf in Turkey. Fragments of the SSU rRNA gene sequences of Cryptosporidium spp. acquired in this study were deposited in GenBank (MN918153-MN918257 and MN918118). Based on a blast search, all of the sequences detected in this study were identified as Cryptosporidium. The nucleotide sequence of $C$. parvum had $99.5 \%$ to $100 \%$ genetic identity with the reference sequences previously published in GenBank (JX298604, KF533079, JQ313985, GQ983351, and MK731971). Among the Cryptosporidium partial SSU rRNA gene sequences, only one sample had $100 \%$ identity with the $C$. bovis reference sequences in GenBank (AB777173, AB628204, EU408317, MK880573) (Fig. 1).

All of the $C$. parvum-positive specimens generated the expected gp60 PCR product. However, only 82 of the 105 isolates the gp60 gene were successfully sequenced and deposited in GenBank (MN962650MN962718, MN998529-MN998541). Three subtypes (IlaA13G4R1, IlaA12G3R1, and IlaA11G3R1) differed from reference sequences regarding the amount of TCA and/or TCG repeats and were considered novel C. parvum subtypes. Eight subtypes were identified among the calf $C$. parvum specimens, including two novel (IlaA12G3R1 and IlaA11G3R1) subtypes and three other lla subtype families (IlaA13G2R1, IlaA15G2R1, and IlaA11G2R1) and three Ild subtype families (IIdA16G1, IIdA18G1, and IIdA22G1). Moreover, six subtypes were identified among the lamb C. parvum specimens, including two novel 
subtypes (IlaA13G4R1 and IlaA12G3R1) and four other lla subtype family (IlaA15G2R1, IlaA13G2R1, IlaA14G3R1, and IlaA11G2R1). In addition, we identified two subtypes (IlaA14G1R1 and IlaA13G2R1) in the goat kid $C$. parvum specimens (Fig. 2 and 3 ).

\section{Discussion}

In the present study, the prevalence of Cryptosporidium was found to be $25.6 \%$, which is consistent with previous studies conducted in Turkey. Except for one calf which was infected with C. bovis, all of the Cryptosporidium from 90 calves, 13 lambs and 2 goat kids were of C. parvum. In Turkey, there is only one study reporting C. bovis in calves in the Mediterranean and Central Anatolia regions [21]. In an another report C. bovis was detected in environmental water in Turkey [35]. Cryptosporidium parvum was detected in a small number of calves and cattle in northeastern Turkey $[23,36]$. C. parvum infections commonly cause profuse watery diarrhea which sometimes contains mucus or blood, dehydration, abdominal pain, loss of appetite, and weight loss. The disease causes severe illness and death in young ruminants. Therefore, Cryptosporidium infections lead to substantial economic losses [1]. Cryptosporidium parvum is not host-specific; accordingly, an environment contaminated with oocysts during an outbreak in calves can give rise to infection in lambs and goat kids that subsequently use the same grazing area.

In Turkey, the prevalence of Cryptosporidium infection in diarrheic calves, lambs, and goat kids has previously been reported to range from 7.5-79.1\% depending on the geographical region or province [17$21,24,36]$. However, most of these studies relied on microscopic examination of fecal specimens. Cryptosporidium infection rates in Europe have been reported as follows: $38.8 \%$ of calves in Italy [3], 37\% and $12 \%$ of dairy and beef calves, respectively, in Belgium [37], 36.7\% of calves in Sweden [38], 22.5\% of calves in Poland [39], 19.2\% of lambs and $37.1 \%$ of goats in Poland [12], $74.4 \%$ of lambs and $93.8 \%$ of goat kids in Spain [11], $13.1 \%$ of lambs and $9.5 \%$ of goat kids in Belgium [40], and $5.1 \%$ of lambs and $7.1 \%$ of goat kids in Greece [10]. From these studies, it is clear that cryptosporidial infections are widespread in calves, lambs, and goat kids in Europe, including Turkey.

In this study, the results of gp60 sequence analysis revealed significant genetic diversity with the presence of two novel subtypes (IlaA12G3R1 and IlaA11G3R1) of the lla subtype family of C. parvum in calves. Most of the calves were found to be infected with subtype llaA13G2R1, which was common subtype in all three hosts and the subtype found firstly in lambs in Turkey. Subtype llaA13G2R1 was previously identified in free-range pre-weaned diarrheic dairy calves and goat kids in Turkey [22]. Moreover, llaA13G2R1 was found the most common subtype in pre-weaned diarrheic calves in the Central Anatolia region of Turkey [21]. This uncommon zoonotic subtype was also reported in Algerian young calves, lambs, and goat kids [41, 42]. Moreover, there are only a few reports of this uncommon subtype in a small number of calves in Belgium, Canada, and the Netherlands [37, 43,44]. This subtype was also detected in a HIV-positive individual in Malaysia [45]. The second-most common subtype identified in this study, IlaA15G2R1 which is the most common subtype found worldwide, has been reported previously in cattle in Kars province of Turkey [24]. This subtype is dominant in humans in Scotland [46]. The high prevalence of subtype llaA15G2R1 in calves and lambs worldwide and its detection in humans suggest 
that it is easily spread among animal populations and readily transmitted to humans as well. Subtype IlaA11G2R1 has not previously been reported in Turkey; however, it has been reported in humans in Slovenia and Slovakia $[47,48]$.

The Ild subtype family (IIdA16G1, IIdA18G1 and IIdA22G1) of C. parvum was also identified in calves in this study. Subtypes IIdA18G1 and IIdA22G1 have previously been detected in calves and goat kids in Turkey [22]. In a study conducted in Greece, IIdA16G1 subtype was identified in diarrheic lambs and goat kids [13]. Within the subtype IId family, IIdA16G1, IIdA18G1 and IIdA22G1 subtypes have previously been reported in calves, lambs, and goat kids in Spain [7]. C. parvum Ild subtypes were found at low frequencies in calves in many European countries [37, 38, 49,50]. IIdA22G1 and IIdA16G1 detected in this study has been identified in humans in Europe $[2,51,52]$.

It was elucidated that $\mathrm{C}$. parvum was the dominant Cryptosporidium species among the calves, lambs and goat kids in the present study. Two novel subtypes (IlaA13G4R1 and IlaA12G3R1) were identified among the lambs. Ila was the dominant subtype family of $\mathrm{C}$. parvum in both lambs and goat kids in this study. Ila and Ild subtypes were found previously in diarrheic goat kids in Turkey [22]. However, the Ild subtype was dominant in lambs and goat kids in northeastern Spain [7]. Interestingly, subtype IlaA14G3R1, identified in lambs in this study was previously found in fresh molluscan shellfish in Italy [53].

Cryptosporidium parvum has been identified in pregnant women, children, and an infant in Turkey, however subtype data are not available [54-56]. Common subtype families of C. parvum are Ila, Ilc, Ild and Ile. The Ila is the predominant family in animals and humans worldwide, whereas Ild is another major zoonotic subtype family in Europe, Asia, North Africa and Australia [57]. The C. parvum subtypes found in this study were previously detected in humans and probably have public health risk.

\section{Conclusions}

The presence of zoonotic C. parvum subtype families (Ila, IId) in this study suggests that calves, lambs, and goat kids are likely to be a major reservoir of C. parvum. Animals infected with these subtypes probably poses a threat to human health in Turkey. Our study showed the diversity of molecularly characterized C. parvum isolates isolated in Turkey; C. bovis was identified in a calf and three novel subtypes (IlaA13G4R1, IlaA11G3R1, and IlaA12G3R1) were found. Further molecular studies in calves, lambs, and goat kids from other provinces are required to better understand the epidemiology of cryptosporidiosis in Turkey.

\section{Abbreviations}

SSU rRNA

Small subunit ribosomal ribonucleic acid; gp60:60 kDa glycoprotein; MZN:Modified Ziehl-Neelsen. 


\section{Declarations}

\section{Acknowledgments}

We would like to thank the staffs of Faculty of Veterinary Medicine, Selcuk University, Turkey for valuable and technical assistance in collection of the specimens.

\section{Funding}

This study was funded by grants-in-aid for Scientific Research (B:17H03913) and (C:16KT0141) and Scientific Research on Innovative Areas (3805) from the Ministry of Education, Culture, Science, Sports, and Technology (MEXT) of Japan, and by a Livestock Promotional Subsidy from the Japan Racing Association.

\section{Availability of data and materials}

All data generated or analyzed during this study are included in this article. The nucleotide sequences generated in this study were deposited in the GenBank database under accession numbers MN918153MN918257, MN918118 and MN962650-MN962718, MN998529-MN998541.

\section{Authors' contributions}

$\mathrm{KK}, \mathrm{FS}$ and $\mathrm{XX}$ were involved in planning the concept. MHBK wrote the manuscript with support from all authors. MHBK and $O C$ manufactured the specimens, performed the experiments and processed the experimental data. MHBK, OC, CC, AAS and HB performed the analysis, designed the figures, organized and sort out all data. MHBK, OC and MIE contributed to the interpretation of the results. KK, FS and XX supervised the findings of this work. All authors read and approved the final manuscript.

\section{Ethics approval}

The research protocol was reviewed and approved by the Research Ethics Committee of Obihiro University of Agricultural and Veterinary Medicine. Permission was obtained from all animal owners before the fecal specimens were collected.

\section{Consent for publication}


Not applicable.

\section{Competing of interests}

The authors have no competing of interests to declare.

\section{References}


1. de Graaf DC, Vanopdenbosch E, Ortega-Mora LM, Abbassi H, Peeters JE. A review of the importance of cryptosporidiosis in farm animals. Int J Parasitol. 1999;29:1269-87.

2. Alves M, Xiao L, Antunes F, Matos O. Distribution of Cryptosporidium subtypes in humans and domestic and wild ruminants in Portugal. Parasitol Res. 2006;99:287-92.

3. Diaz P, Varcasia A, Pipia AP, Tamponi C, Sanna G, Prieto A, et al. Molecular characterization and risk factor analysis of Cryptosporidium spp. in calves from Italy. Parasitol Res.

2018;117:3081-90.

4. Olson ME, O'Handley RM, Ralston BJ, McAllister TA, Thompson RC. Update on Cryptosporidium and Giardia infections in cattle. Trends Parasitol. 2004;20:185-91.

5. Xiao L, Feng Y. Zoonotic cryptosporidiosis. FEMS Immunol Med Microbiol. 2008;52:309-23.

6. Wells B, Thompson S. Cryptosporidiosis in cattle. The Moredun foundation news sheet. 2014;6:1

7. Quilez J, Torres E, Chalmers RM, Hadfield SJ, Del Cacho E, Sanchez-Acedo C.

Cryptosporidium genotypes and subtypes in lambs and goat kids in Spain. Appl Environ Microbiol. 2008b;74:6026-31.

8. Rieux A, Paraud C, Pors I, Chartier C. Molecular characterization of Cryptosporidium spp. in pre-weaned kids in a dairy goat farm in western France. Vet Parasitol. 2013;192:268-72.

9. $\quad$ Mi R, Wang X, Huang Y, Zhou P, Liu Y, Chen $Y$, et al. Prevalence and molecular characterization of Cryptosporidium in goats across four provincial level areas in China. PLoS One. 2014;9:e111164.

10. Tzanidakis N, Sotiraki S, Claerebout E, Ehsan A, Voutzourakis N, Kostopoulou D, et al. Occurrence and molecular characterization of Giardia duodenalis and Cryptosporidium spp. in sheep and goats reared under dairy husbandry systems in Greece. Parasite. 2014;21:45.

11. Diaz P, Quilez J, Prieto A, Navarro E, Perez-Creo A, Fernandez G, et al. Cryptosporidium species and subtype analysis in diarrheic pre-weaned lambs and goat kids from northwestern Spain. Parasitol Res. 2015;114:4099-105.

12. Kaupke A, Michalski MM, Rzeżutka A. Diversity of Cryptosporidium species occurring in sheep and goat breeds reared in Poland. Parasitol Res. 2017;116:871-79.

13. Papanikolopoulou V, Baroudi D, Guo Y, Wang Y, Papadopoulos E, Lafi SQ, et al. Genotypes and subtypes of Cryptosporidium spp. in diarrheic lambs and goat kids in northern Greece. Parasitol Int. 2018;67:472-75.

14. Ryan U, Fayer R, Xiao L. Cryptosporidium species in humans and animals: current understanding and research needs. Parasitology. 2014;141:1667-85.

15. Chalmers RM, Elwin K, Reilly WJ, Irvine H, Thomas AL, Hunter PR. Cryptosporidium in farmed animals: the detection of a novel isolate in sheep. Int J Parasitol. 2002;32:21-26.

16. Mueller-Doblies D, Giles M, Elwin K, Smith RP, Clifton-Hadley FA, Chalmers RM. Distribution of Cryptosporidium species in sheep in the UK. Vet Parasitol. 2008;154:214-19.

17. Cicek M, Korkoca H, Gul A. Investigation of Cryptosporidium spp. in workers of the Van municipality slaughterhouse and in slaughtered animals. Turkiye Parazitol Derg. 2008;32:811.

18. Sari B, Arslan MO, Gicik Y, Kara M, Tasci GT. The prevalence of Cryptosporidium species in diarrhoeic lambs in Kars province and potential risk factors. Trop Anim Health Prod. 
19. Sari B, Aktas MS, Arslan MO. The prevalence of Cryptosporidium spp. in calves in Erzurum province. Turkiye Parazitol Derg. 2008;32:116-19.

20. Gul A, Cicek M, Kilinc O. Prevalence of Eimeria spp., Cryptosporidium spp. and Giardia spp. in calves in the Van province. Turkiye Parazitol Derg. 2008;32:202-4.

21. Yildirim A, Adanir R, Inci A, Yukari BA, Duzlu O, Onder Z, et al. Prevalence and genotyping of bovine Cryptosporidium species in the mediterranean and central Anatolia region of Turkey. Comp Immunol Microbiol Infect Dis. 2020;69:101425.

22. Taylan-Ozkan A, Yasa-Duru S, Usluca S, Lysen C, Ye J, Roellig DM, et al. Cryptosporidium species and Cryptosporidium parvum subtypes in dairy calves and goat kids reared under traditional farming systems in Turkey. Exp Parasitol. 2016;170:16-20.

23. Tanriverdi S, Markovics A, Arslan MO, Itik A, Shkap V, Widmer G. Emergence of distinct genotypes of Cryptosporidium parvum in structured host populations. Appl Environ Microbiol. 2006;72:2507-13.

24. Arslan MO, Ekinci Al. Determination of Cryptosporidium parvum subtypes in cattle in Kars province of Turkey. Kafkas Univ Vet Fak Derg. 2012;18:A221-26.

25. Casemore DP. ACP Broadsheet 128: Laboratory methods for diagnosing cryptosporidiosis. J Clin Pathol. 1991;44:445-51.

26. McLauchlin J, Pedraza-Díaz S, Amar-Hoetzeneder C, Nichols GL. Genetic characterization of Cryptosporidium strains from 218 patients with diarrhea diagnosed as having sporadic cryptosporidiosis. J Clin Microbiol. 1999;37:3153-58.

27. Sulaiman IM, Lal AA, Xiao L. Molecular phylogeny and evolutionary relationships of Cryptosporidium parasites at the actin locus. J Parasitol. 2002;88:388-94.

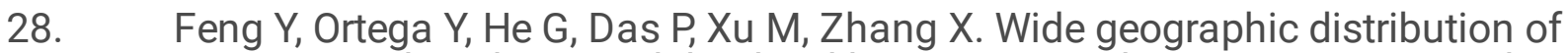
Cryptosporidium bovis and the deer like genotype in bovines. Vet Parasitol. 2007;144:1-9.

29. Sulaiman IM, Hira PR, Zhou L, Al-Ali FM, Al-Shelahi FA, Shweiki HM, et al. Unique endemicity of cryptosporidiosis in children in Kuwait. J Clin Microbiol. 2005;43:2805-9.

30. Larkin MA, Blackshields G, Brown NP, Chenna R, McGettigan PA, McWilliam H, et al. Clustal W and Clustal X Version 2.0. Bioinformatics. 2007;23:2947-48.

31. Hall TA. BioEdit: a user-friendly biological sequence alignment editor and analysis program for windows 95/98/NT. Nucl Acids Symp Ser. 1999;41:95-98.

32. Kumar S, Stecher G, Tamura K. Molecular evolutionary genetics analysis version 7.0 for bigger datasets. Mol Biol Evol. 2016;33:1870-74.

33. Tamura K. Estimation of the number of nucleotide substitutions when there are strong transition-transversion and G+C-content biases. Mol Biol Evol. 1992;9:678-87.

34. Hasegawa M, Kishino H, Yano T. Dating of the human-ape splitting by a molecular clock of mitochondrial DNA. J Mol Evol. 1985;22:160-74.

35. Koloren Z, Ayaz E. Genotyping of Cryptosporidium spp. in environmental water in Turkey. Acta Parasitol. 2016;61:671-79.

36. Gunduz N, Arslan MO. Determining the prevalence of Cryptosporidium infections with acid fast staining and ELISA in calves at the Kars province of Turkey. Turkiye Parazitol Derg. 
2017;41:5-8.

37. Geurden T, Berkvens D, Martens C, Casaert S, Vercruysse J, Claerebout E. Molecular epidemiology with subtype analysis of Cryptosporidium in calves in Belgium. Parasitology. 2007;134:1981-87.

38. Björkman C, Lindström L, Oweson C, Ahola $H$, Troell $K$, Axén C.

Cryptosporidium infections in suckler herd beef calves. Parasitology. 2015;142:1108-14.

39. Kaupke A, Rzeżutka A. Emergence of novel subtypes of Cryptosporidium parvum in calves in Poland. Parasitol Res. 2015;114:4709-16.

40. Geurden T, Thomas P, Casaert S, Vercruysse J, Claerebout E. Prevalence and molecular characterization of Cryptosporidium and Giardia in lambs and goat kids in Belgium. Vet Parasitol. 2008;155:142-45.

41. Baroudi D, Hakem A, Adamu H, Amer S, Khelef D, Adjou K, et al. Zoonotic Cryptosporidium species and subtypes in lambs and goat kids in Algeria. Parasit Vectors. 2018;11:582.

42. Benhouda D, Hakem A, Sannella AR, Benhouda A, Cacciò SM. First molecular investigation of Cryptosporidium spp. in young calves in Algeria. Parasite. 2017;24:15.First molecular investigation of Cryptosporidium spp. in young calves in Algeria

43. Trotz-Williams LA, Martin DS, Gatei W, Cama V, Peregrine AS, Martin SW, et al. Genotype and subtype analyses of Cryptosporidium isolates from dairy calves and humans in Ontario. Parasitol Res. 2006;99:346-52.

44. Wielinga PR, de Vries A, van der Goot TH, Mank T, Mars MH, Kortbeek LM. Molecular epidemiology of Cryptosporidium in humans and cattle in the Netherlands. Int J Parasitol. 2008;38:809-17.

45. Iqbal A, Lim LA, Surin J, Sim BL. High diversity of Cryptosporidium subgenotypes identified in Malaysian HIV/AIDS individuals targeting gp60 gene. PLoS One. 2012;7:e31139.

46. Deshpande AP, Jones BL, Connelly L, Pollock KG, Brownlie S, Alexander CL. Molecular characterization of Cryptosporidium parvum isolates from human cryptosporidiosis cases in Scotland. Parasitology. 2015;142:318-25.

47. Soba B, Logar J. Genetic classification of Cryptosporidium isolates from humans and calves in Slovenia. Parasitology. 2008;135:1263-70.

48. Hatalová E, Valenčáková A, Luptáková L, Špalková M, Kalinová J, Halánová M, et al. The first report of animal genotypes of Cryptosporidium parvum in immunosuppressed and immunocompetent humans in Slovakia. Transbound Emerg Dis. 2019;66:243-49.

49. Plutzer J, Karanis P. Genotype and subtype analyses of Cryptosporidium isolates from cattle in Hungary. Vet Parasitol. 2007;146:357-62.

50. Broglia A, Reckinger S, Caccio SM, Nockler K. Distribution of Cryptosporidium parvum subtypes in calves in Germany. Vet Parasitol. 2008;154:8-13.

51. Vieira PM, Mederle N, Lobo ML, Imre K, Mederle O, Xiao L, et al. Molecular characterization of Cryptosporidium (Apicomplexa) in children and cattle in Romania. Folia Parasitol. 2015;62.

52. de Lucio A, Merino FJ, Martinez-Ruiz R, Bailo B, Aguilera M, Fuentes I, et al. Molecular genotyping and sub-genotyping of Cryptosporidium spp. isolates from symptomatic individuals attending two major public hospitals in Madrid, Spain. Infect Genet Evol. 2016;37:49-56. 
53. Giangaspero A, Papini R, Marangi M, Koehler AV, Gasser RB. Cryptosporidium parvum genotype lla and Giardia duodenalis assemblage A in Mytilus galloprovincialis on sale at local food markets. Int J Food Microbiol. 2014;171:62-67.

54. Hizli S, Aksoy U, Arslan N, Usluca S, Bekem O, Yalcin G, et al. Cryptosporidium parvum diarrhea in an infant with short bowel syndrome. J Pediatr Surg. 2006;41:e5-6.

55. Tamer GS, Turk M, Dagci H, Pektas B, Guy EC, Guruz AY, et al. The prevalence of cryptosporidiosis in Turkish children, and genotyping of isolates by nested polymerase chain reaction-restriction fragment length polymorphism. Saudi Med J. 2007;28:1243-46.

56. Dogan N, Saglik I. Cyclospora cayetanensis and Cryptosporidium parvum coinfection in a pregnant women with prolonged diarrhea. Mikrobiyol Bul. 2010;44:155-59.

57. Wang R, Zhang L, Axen C, Bjorkman C, Jian F, Amer S, et al. Cryptosporidium parvum Ild family: clonal population and dispersal from western asia to other geographical regions. Sci Rep. 2014;4:4208.

Figures

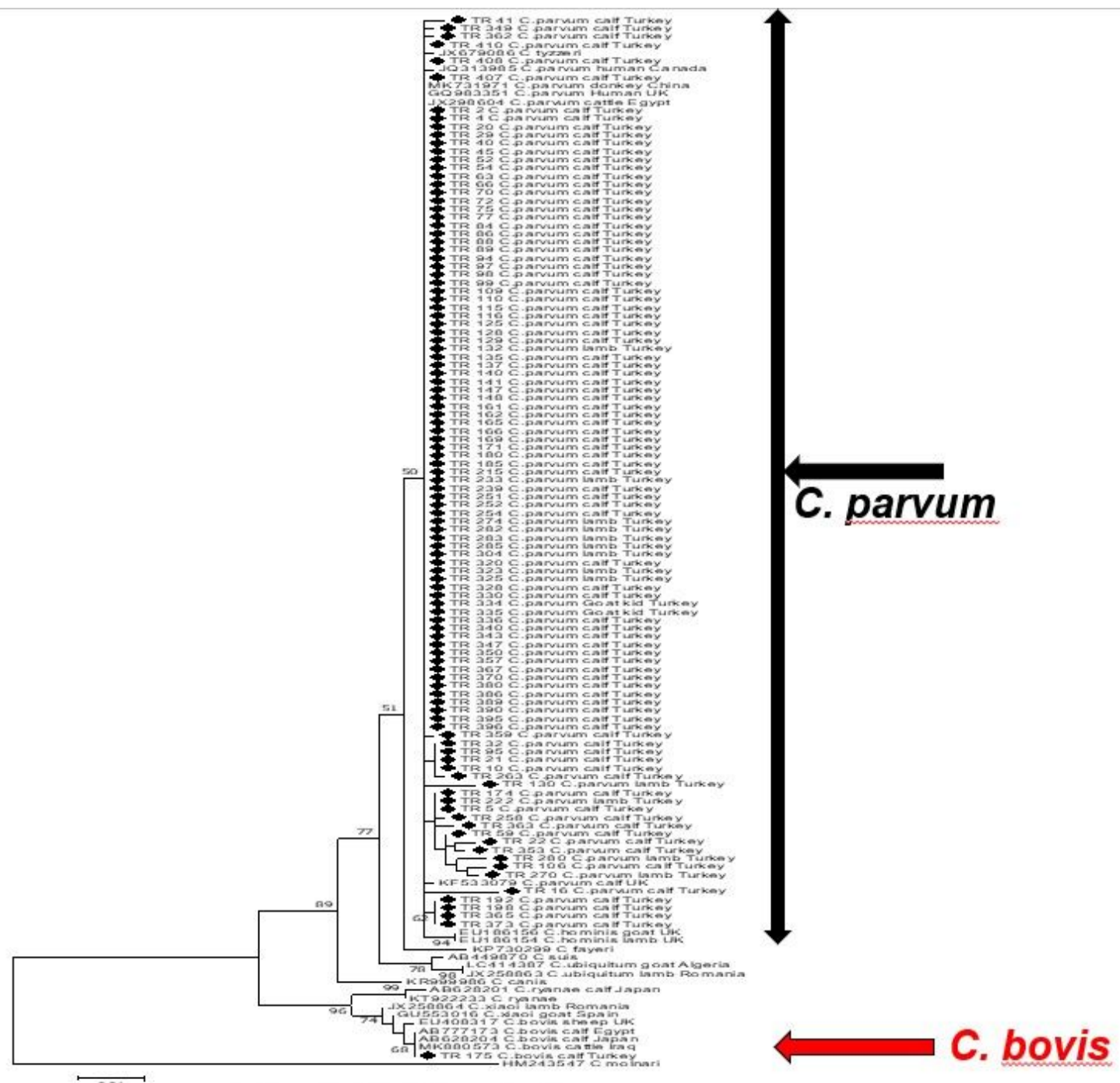

Fig. 1 Kabir et al.

Figure 1 
Phylogenetic tree based on partial sequences of the SSU rRNA genes for Cryptosporidium spp. The phylogenetic tree was constructed without nucleotide gaps by using a Maximum Likelihood analysis with 1000 replicates based on the T92+G model. Cryptosporidium molnari sequence was used as the outgroup. Only bootstrap values $>50 \%$ from 1000 replicates are shown at the nodes. Black filled bold indicate sequences generated in the present study

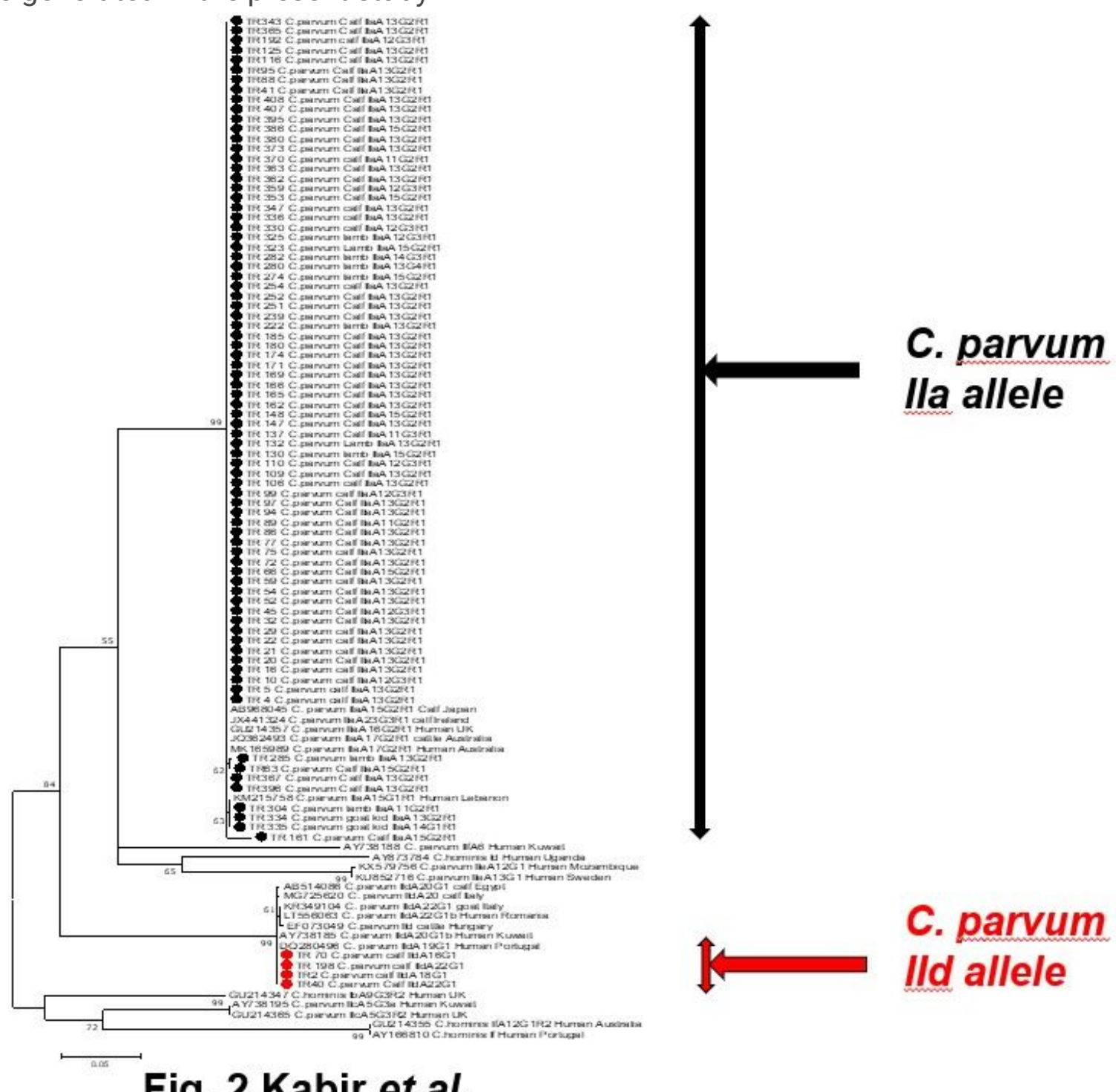

Fig. 2 Kabir et al.

Figure 2

Phylogenetic tree based on partial sequences of the gp60 genes for Cryptosporidium parvum. The phylogenetic tree was constructed without nucleotide gaps by using a Maximum Likelihood analysis with 1000 replicates based on the Hasegawa-Kishino-Yano model. Only bootstrap values $>50 \%$ from 1000 replicates are shown at the nodes. Black and Red filled circles represent sequences in two different subtype families generated in this study 


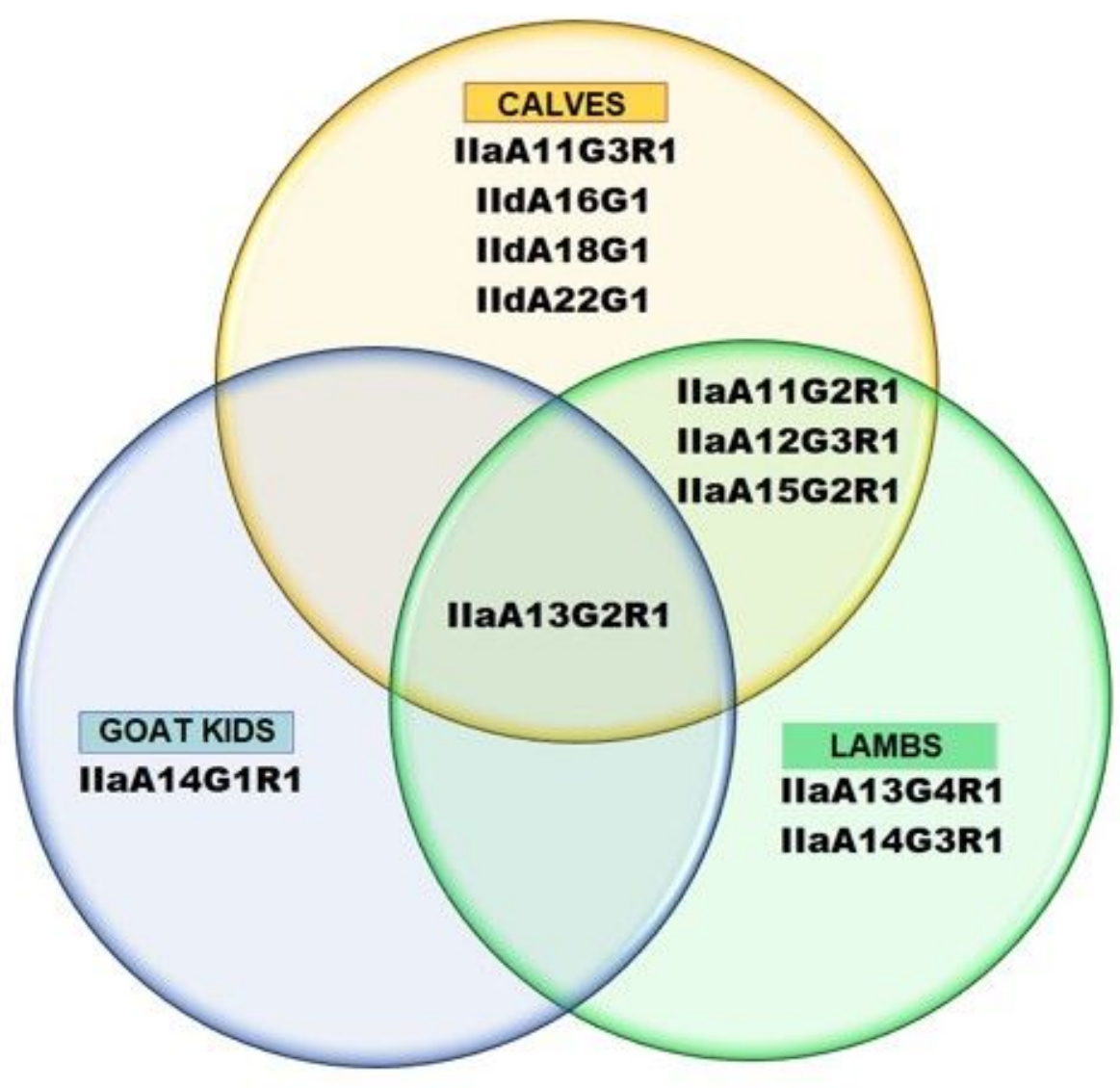

Fig. 3 Kabir et al.

Figure 3

Distribution of subtypes in the diarrheic calves, lambs and goat kids

\section{Supplementary Files}

This is a list of supplementary files associated with this preprint. Click to download.

- Graphicalabstract.pptx

- Additionalfile.docx 